\title{
MtENOD11 Gene Activation During Rhizobial Infection and Mycorrhizal Arbuscule Development Requires a Common AT-Rich-Containing Regulatory Sequence
}

\author{
Aurélien Boisson-Dernier, Andry Andriankaja, Mireille Chabaud, Andreas Niebel, Etienne-P. Journet, \\ David G. Barker, and Fernanda de Carvalho-Niebel
}

Laboratory of Plant Microbe Interactions (LIPM), CNRS-INRA, BP 52627, 31326 Castanet-Tolosan Cedex, France

Submitted 20 May 2005. Accepted 7 July 2005.

\begin{abstract}
The MtENOD11 gene from the model legume Medicago truncatula is transcriptionally activated both in response to Sinorhizobium meliloti Nod factors and throughout infection of root tissues by the nitrogen-fixing microsymbiont. To identify the regulatory sequences involved in symbiosisrelated MtENOD11 expression, a series of promoter deletions driving the $\beta$-glucuronidase reporter gene were analyzed in transgenic $M$. truncatula roots. These studies have revealed that distinct regulatory regions are involved in infection-related MtENOD11 expression compared with preinfection (Nod factor-mediated) expression. In particular, the 257-bp promoter sequence immediately upstream from the start codon is sufficient for infection-related expression, but is unable to drive gene transcription in response to the Nod factor elicitor. This truncated promoter is also sufficient to confer MtENOD11 expression during both the arbuscular mycorrhizal (AM) association and the parasitic interaction with root-knot nematodes. Site-directed mutagenesis further showed that a previously identified nodule-specific AT-rich motif is required for high-level MtENOD11 expression during $S$. meliloti infection as well as during AM colonization. However, mutation of this motif does not affect gene expression associated with nematodefeeding sites. Taken together, these results suggest a close link between regulatory mechanisms controlling transcriptional early nodulin gene activation during both rhizobial and $\mathrm{AM}$ root endosymbioses.
\end{abstract}

Additional keywords: AATAA motif, Agrobacterium rhizogenes, Glomus, Meloidogyne, nodFnodL.

Legumes are able to establish an endosymbiotic association with soil bacteria known collectively as rhizobia. This interacttion leads to the formation of a novel plant organ, the "root nodule", in which bacteria reduce atmospheric nitrogen to ammonia, thus providing the host plant with a privileged source of combined nitrogen. During the early steps of nodulation, the

A. Boisson-Dernier and A. Andriankaja have contributed equally to this work.

Corresponding author: F. de Carvalho-Niebel;

E-mail: fniebel@toulouse.inra.fr

Present address of A. Boisson-Dernier: Division of Biological Sciences, University of California, San Diego, La Jolla, CA 92093-0116, U.S.A.

Gene sequence data for MtENOD11 is available in the EMBL database under accession number AJ297721. bacteria induce curling of epidermal root hairs and enter the root via membrane invaginations called infection threads formed within the root hair curl (Gage 2004). These infection threads then grow toward the nodule primordium, formed from mitotically activated cortical cells in inner root tissues. Bacteria are then released from the infection thread into cells of the primordium, resulting several days later in the development of the symbiotic nitrogen-fixing nodule.

Specific lipo-chitooligosaccharide signal molecules secreted by Rhizobium spp., called Nod factors (NFs), play a pivotal role in successful host-bacteria recognition and subsequent root nodulation (Limpens and Bisseling 2003; Oldroyd et al. 2005). Genetic and molecular studies in the model legumes Lotus japonicus and Medicago truncatula have identified several genes required for nodule development and for activation of early NF responses such as calcium spiking, root hair deformation, cortical cell divisions, and the transcriptional activation of a number of plant genes known as early nodulin genes (D'Haeze and Holsters 2002; Oldroyd et al. 2005).

The M. truncatula ENOD11 gene, encoding a putative cellwall-repetitive (hydroxy) proline-rich protein (RPRP), is one of the earliest known marker genes induced in the root epidermis following NF application (Charron et al. 2004). MtENOD11 activation occurs rapidly ( 1 to $3 \mathrm{~h}$ ) following exogenous NF addition and closely resembles the activation of the gene in root hairs within the first hours following Sinorhizobium meliloti inoculation and before bacterial infection (Charron et al. 2004; Journet et al. 2001). This Nod factor-mediated preinfection response is observed in epidermal tissues lying behind the root tip and corresponds to the region of root hair emergence and development where successful infections will occur 36 to $48 \mathrm{~h}$ later. In addition to this rapid preinfection response, MtENOD11 expression is also observed at a later stage specifically associated with the rhizobial infection process itself in both root and nodule tissues (Journet et al. 2001). Although the more generalized preinfection expression of MtENOD11 in the epidermis can be mimicked by purified Nod factors, the subsequent localized expression of the gene in root hairs and cortical cells is strictly correlated with the formation of the infection thread.

In addition to being a marker gene for the Rhizobium interaction, MtENOD11 is also activated during the arbuscular mycorrhizal (AM) endosymbiotic association (Chabaud et al. 2002; Journet et al. 2001). Genetic evidence suggests that common signaling pathways for AM establishment are shared with the nitrogen-fixing symbiosis (Kistner and Parniske 2002). Furthermore, several genes other than MtENOD11 are expressed during both AM and rhizobial interactions (Albrecht et 
al. 1998; Hohnjec et al. 2003; Journet et al. 2001; Manthey et al. 2004; Vieweg et al. 2004, 2005). However, it is not yet known whether common mechanisms are shared in the transcriptional activation of these genes during nodulation and endomycorrhization.

To date, functional promoter studies have been conducted extensively with so-called "late" nodulin genes, which are strongly expressed in mature nitrogen-fixing nodules. This has led to the identification of the nodule-specific conserved motifs AAAGAT and CTCTT (Andersson et al. 1997; Stougaard 1987). These nodule-specific motifs are not found in the promoters of early nodulin genes, such as the pea ENOD12 gene. On the other hand, it has been shown that an AT-rich motif (TTATT(N) $)_{7-12}$ AATAA), is required for conferring high expression levels of PSENOD12 in the early symbiotic nodule zone and in cells of the nodule primordium (Hansen et al. 1999; Vijn et al. 1995). Despite this, regulatory sequences have not yet been identified for any early nodulin gene transcriptionally activated in the root epidermis either in response to Nod factor or during rhizobial infection.

In order to identify the regulatory sequences involved in MtENOD11 gene activation during endosymbiotic associations, we have performed a detailed functional analysis of the MtENOD11 promoter using Agrobacterium rhizogenes-transformed M. truncatula plants (Boisson-Dernier et al. 2001; Journet et al. 2001). Our experiments have revealed that distinct promoter regions are involved in the preinfection (NF-mediated) and infection-related expression of the MtENOD11 gene. Interestingly, the promoter region required for infection-related expression, lying within the 257-bp sequence upstream of the ATG start codon, is also sufficient to regulate gene expression during AM colonization. Furthermore, site-directed mutagenesis has shown that the AT-rich motif identified by Hansen and associates (1999) is required for efficient MtENOD11 expression during both rhizobial infection and endomycorrhizal colonization. The fact that this motif does not appear to be important for expression in other contexts, such as lateral root initiation and the development of nematode-feeding sites, underlines the specificity of this motif for regulated gene expression during endosymbiotic rhizobial and AM associations.

\section{RESULTS}

Distinct promoter regions control MtENOD11 expression during preinfection and infection stages of the $S$. meliloti interaction.

Using the $\beta$-glucuronidase $(G U S)$ reporter gene, we previously have shown that a $2.3-\mathrm{kb}$ MtENOD11 promoter sequence is sufficient to confer both symbiotic and nonsymbiotic expression of the gene in either transgenic plants or transgenic roots generated by A. rhizogenes transformation (Boisson-Dernier et al. 2001; Journet et al. 2001). To identify regulatory sequences involved in MtENOD11 expression prior to and throughout $S$. meliloti infection, we first generated a $5^{\prime}$ deletion series based on the 2.3-kb MtENOD11 promoter transcriptionally fused to the $G U S$ reporter gene (Fig. 1). After cloning into a binary vector (discussed below), these constructs were introduced into the A. rhizogenes strain ARqua1, which then was used to generate transformed roots on $M$. truncatula "composite" plants (Boisson-Dernier et al. 2001). In parallel with the analysis of expression in response to $S$. meliloti inoculation and NF treatment, GUS reporter activity was also evaluated in lateral root primordia (Journet et al. 2001). This nonsymbiotic expression in root primordia serves as a useful internal control, because it is not influenced either by $S$. meliloti inoculation or $\mathrm{NF}$ treatments.

To study the early preinfection (Nod factor-mediated) expression which occurs prior to root hair curling and infection thread formation, transgenic roots of composite plants harboring the different MtENOD11 promoter deletion constructs were stained for GUS activity either $24 \mathrm{~h}$ postinoculation with $S$. meliloti or following $16 \mathrm{~h}$ of treatment with a $10^{-9} \mathrm{M}$ aque-

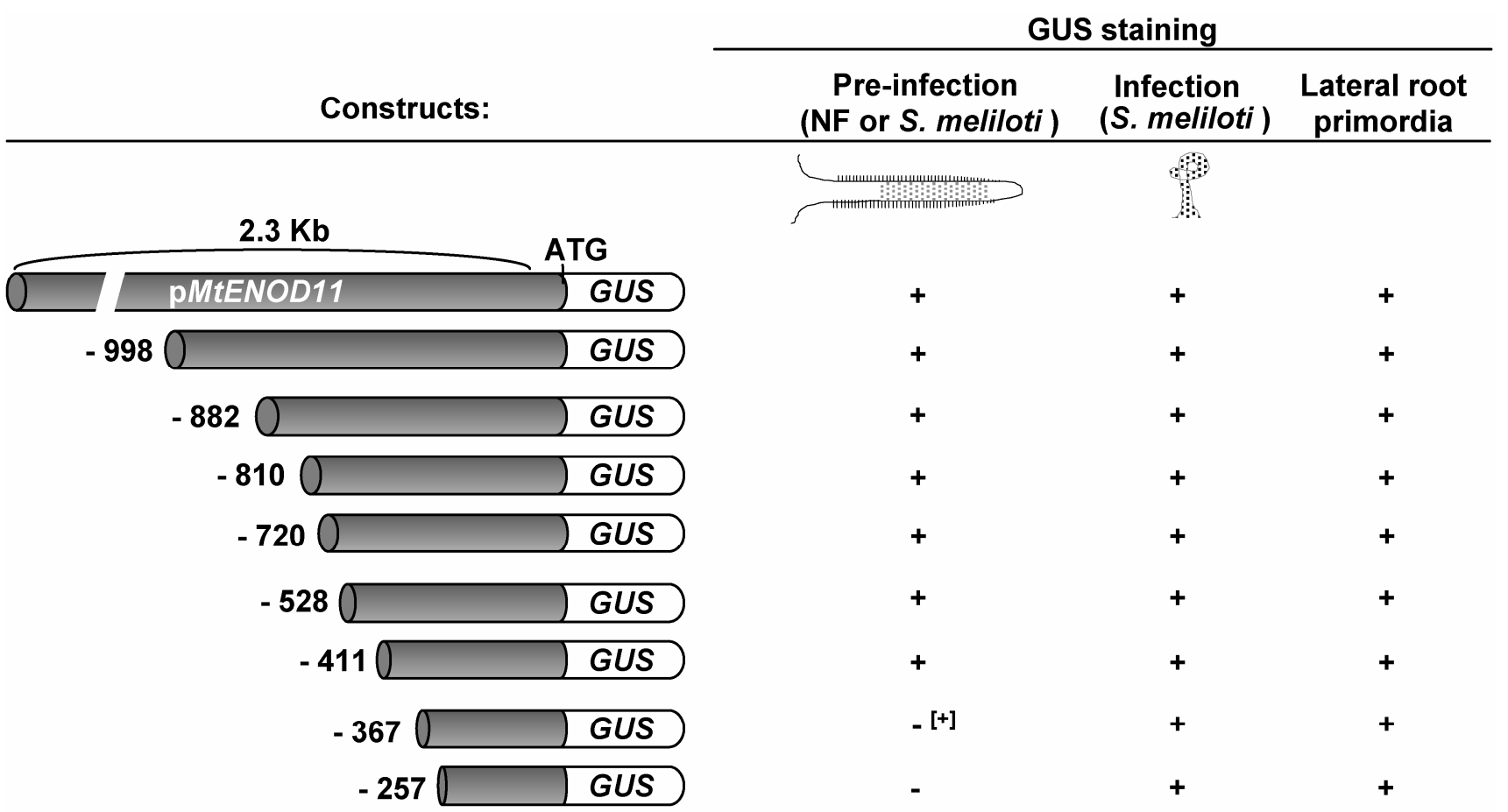

Fig. 1. Schematic diagram of MtENOD11 promoter deletion constructs and the corresponding $\beta$-glucuronidase (GUS) staining analyses in both symbiotic (Nod factor addition and Sinorhizobium meliloti inoculation) and nonsymbiotic (lateral root primordia) contexts. The positions of promoter deletions are indicated in relation to the ATG start codon. At least 60 independent transgenic roots were analyzed for each construct and the overall GUS expression in each case is denoted by + (moderate to strong tissue-specific activity), $-^{[+]}$(weak, residual activity), and - (undetectable staining). 
ous solution of purified NFs. Our results (Fig. 1) show that deletions down to position -411 relative to the ATG initiation codon drive NF- or $S$. meliloti-induced GUS expression in the root epidermis (Fig. 2A). In control experiments, epidermalspecific GUS staining was never observed in roots that were either treated with water or inoculated with a $S$. meliloti nodA mutant defective in NF synthesis (data not shown). This underlines the essential role of NFs in the triggering of this epidermal response by the microsymbiont. Additional 5' deletions down to -367 and -257 resulted in either a major reduction or complete abolition, respectively, of the NF-mediated expression in the root epidermis (Figs. 1 and 2B). The nonsymbiotic expression of these two deletions in lateral root primodia is not affected (Fig. 1); therefore, these results imply that cis-elements specifically involved in the NF-dependent activation of the MtENOD11 gene in the root epidermis are located between positions -411 and -257 of the promoter. These findings also confirm that the rapid induction of MtENOD11 expression in the root epidermis in response to either purified NFs or $S$. meliloti inoculation is mediated by the same promoter region, in line with the fact that NFs are sufficient to activate the MtENOD11 preinfection response.

The identical deletion series then was tested with respect to expression throughout $S$. meliloti infection by analyzing GUS reporter activity in transgenic roots 3,4 , and 5 days postinoculation (dpi). Interestingly, in contrast to the NF-mediated re- sponse, all deletions down to -257 were able to confer $S$. meliloti-induced expression in root infection sites (Figs. 1 and $2 \mathrm{~A}$ through D). Furthermore, analysis of the -257 deletion construct in nitrogen-fixing nodules revealed that this shortened promoter region is also sufficient to direct $G U S$ expression in the distal infection zone of the nodule (Fig. 2E), similarly to the full-length MtENOD11 promoter. Taken together, these results led us to conclude that regulatory sequences involved in the Nod factor-mediated preinfection and infection-related expression of the MtENOD11 gene are located in distinct promoter regions. They also imply that different regulatory mechanisms are probably involved in driving $M t E N O D 11$ expression during these two early phases of the plant-bacteria interaction.

\section{Infection-associated activation}

\section{of MtENOD11 precedes infection thread formation.}

In the above experiments, localized expression of the -257 $\mathrm{p} M t E N O D 11$ deletion was observed in S. meliloti-infected root hairs and neighboring cortical cells. To determine whether this infection-associated expression actually requires infection thread formation in the root hair, we made use of the infectiondefective $S$. meliloti double mutant nodFnodL (Ardourel et al. 1994; Catoira et al. 2000). This mutant elicits root hair curling but no infection threads are initiated from within the curl. Even in the absence of infection, the -257 deletion can drive GUS expression in the highly deformed root hairs formed fol-

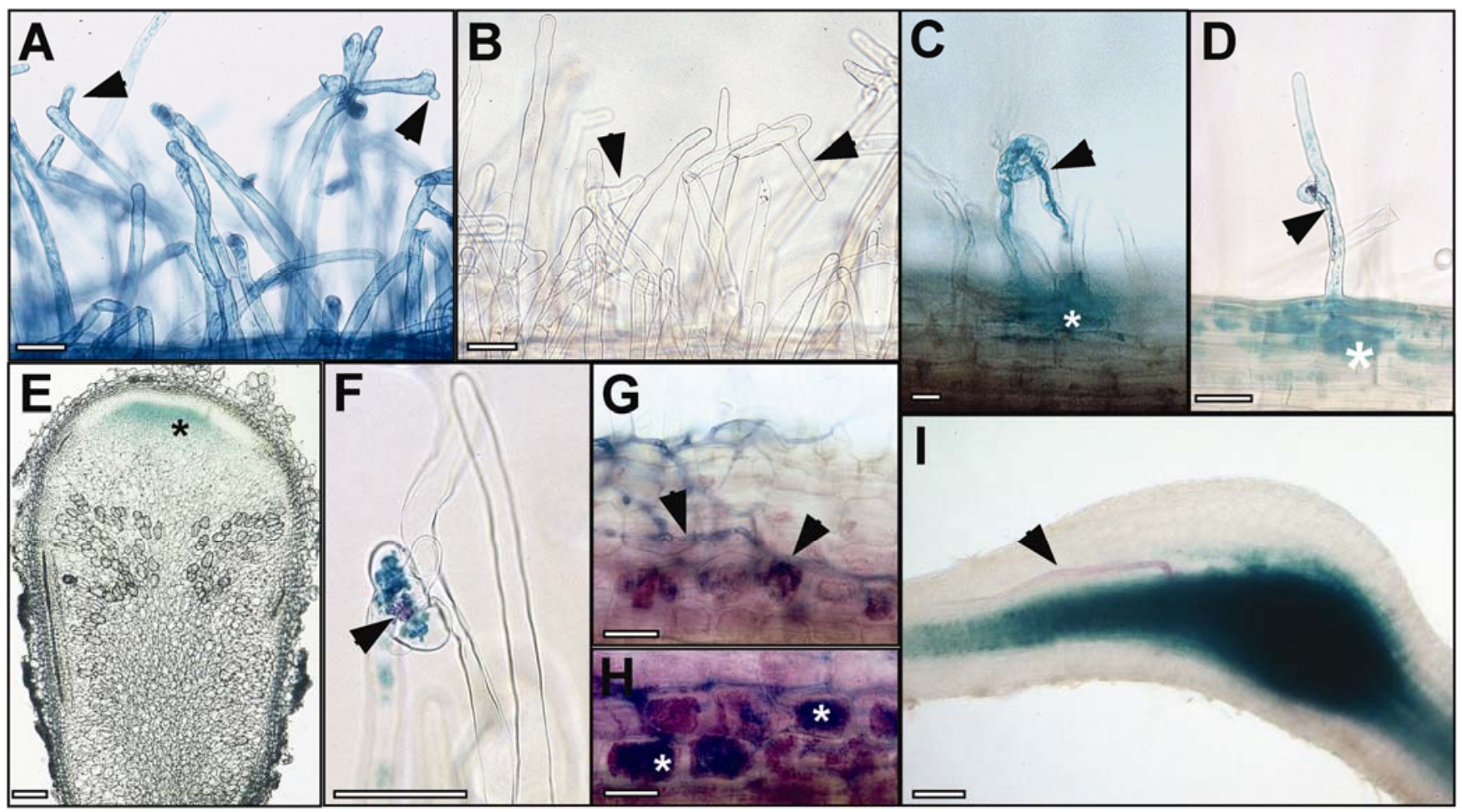

Fig. 2. Histochemical localization of $\beta$-glucuronidase (GUS) activity in Agrobacterium rhizogenes-transformed Medicago truncatula roots expressing the -411 and -257 MtENOD11 promoter deletion constructs. A and $\mathbf{B}$, Root hairs on transgenic roots carrying the $\mathbf{A},-411$ and $\mathbf{B},-257$ deletion constructs driving GUS reporter that have been treated for $16 \mathrm{~h}$ with a $10^{-9} \mathrm{M}$ solution of Nod factors (NFs). Arrows indicate root hair deformations characteristics of NF treatment. $\mathbf{C}$ and $\mathbf{D}$, Sinorhizobium meliloti infection in root hairs 3 days postinoculation (dpi) of transgenic roots expressing either the $\mathbf{C},-411$ or $\mathbf{D},-257$ promoter deletion constructs. Infection threads (arrows) are visualized in red (Magenta-gal) after histochemical staining for the constitutive $\beta$-galactosidase activity. GUS reporter expression in blue (X-gluc) is observed both in root hairs containing infection threads (arrows) and in adjacent outer cortical cells (asterisks) in front of the invading infection thread. E, Section of a 20-day-old mature nitrogen-fixing nodule ( $80 \mu \mathrm{m}$ thick) expressing the $-257 \mathrm{deletion}$ construct. GUS activity (blue) is localized in the distal nodule invasion zone II (asterisk) just below the nodule meristematic zone. F, Epidermal root hair expressing the -257 construct 5 dpi with the $S$. meliloti nodFnodL mutant strain. GUS expression (blue) is present in a highly deformed root hair encircling a swollen bacterial microcolony (in red, arrow) without formation of infection threads. $\mathbf{G}$ and $\mathbf{H}$, Transgenic roots expressing the -257 construct driving GUS 20 dpi with Glomus intraradices. GUS activity (in red) is detected in G, colonized arbuscular mycorrhizal (AM) roots (arrows indicate intraradicular colonizing AM hyphae) and $\mathbf{H}$, associated with arbuscule-containing inner cortical cells (asterisks). I, Transgenic root expressing the -257 construct 5 dpi with Meloidogyne incognita larvae (arrow). GUS staining (blue) is associated with pericycle-derived cells surrounding a nematode feeding site. Bars $=50$ $\mu \mathrm{m}$ (A through D, F, G, and H) or $150 \mu \mathrm{m}$ (E and I). 
lowing inoculation with the $S$. meliloti nodFnodL mutant (Fig. 2F). Because preinfection NF-dependent activation of MtENOD11 is totally absent in the -257 deletion construct (Fig. 2B), this result clearly shows that the specific expression of MtENOD11 associated with root infection does not require infection thread formation.

The -257 MtENOD11 truncated promoter is sufficient for gene activation in AM and nematode-infected roots.

The 2.3-kb pMtENOD11-GUS gene fusion was previously shown to be transcriptionally activated in $M$. truncatula roots during different stages of root colonization by AM fungi (Chabaud et al. 2002; Journet et al. 2001). This activation is associated with AM infection of both epidermal and cortical tissues, as well as during arbuscule differentiation in the inner cortex. To determine whether similar MtENOD11 promoter sequences regulate expression during $S$. meliloti infection and AM colonization, we analyzed the expression of the -257 deletion fused to the GUS reporter in transgenic roots colonized by the AM fungus Glomus intraradices. Our results show that the -257 deletion is sufficient for GUS reporter expression in cortical cells associated with developing arbuscules (Fig. 2G and $\mathrm{H}$ ). Therefore, we conclude that the 257-bp region contains the regulatory sequences necessary for MtENOD11 expression during the AM-symbiotic association.

Certain early nodulin genes such as MtENOD40 are not only expressed during endosymbiotic root associations but also during plant interactions with the root knot nematode Meloidogyne incognita (Favery et al. 2002; Koltai et al. 2001). To examine whether this is also the case for the MtENOD11 gene, we analyzed the expression of GUS fusions driven by either the entire $2.3-\mathrm{kb}$ promoter fragment or the -257 deletion in roots colonized by the $M$. incognita nematode. No activation of either GUS fusion was detected during early stages of nematode penetration and progression through different root tissues (not shown). However, histochemical GUS activity was detected for both constructs exclusively in nematode-induced galls, and particularly in pericycle-derived tissues surrounding the developing giant cells which form the nematode feeding sites (Fig. 2I) (Bird 2004; Davis et al. 2004). Strong GUS activity was observed as soon as galls and feeding sites could be observed (3 to 4 dpi) and then gradually declined at later timepoints (7 dpi) (data not shown). Thus, we can conclude that the MtENOD11 gene is also transcriptionally activated during the association with root knot nematodes, and our results show that the 257-bp promoter region is sufficient to confer this expression.
The AATAA motif

within the 257-bp MtENOD11 promoter region

is important for endosymbiotic but not

for nematode-related gene expression.

As stated earlier, Hansen and associates (1999) were able to show, in a heterologous system, that the AT-rich motif (TTATT $(\mathrm{N})_{7-12}$ AATAA) present within the first $200 \mathrm{bp}$ of the PSENOD12B promoter is essential for high-level gene expression in nodules. Mutations introduced within this motif, and especially modifying the AATAA sequence, led to a major reduction in gene expression in nodule tissues. Interestingly, this AT-rich motif is well conserved in a number of early nodulin gene promoters (Rodriguez-Llorente et al. 2003; Vijn et al. 1995), including the 257-bp promoter region of MtENOD11 (Fig. 3). As a first step toward evaluating the importance of the AATAA motif with respect to MtENOD11 expression, we examined the effect of two additional $5^{\prime}$ deletions (-195 and -146) located upstream and downstream, respectively, of the AT-rich motif (Fig. 3). Whereas no clear difference in rhizobial infection-related GUS activity was found between the -257 and -195 deletion constructs, significantly reduced GUS expression levels were observed for the shorter -146 promoter deletion (data not shown). This result is consistent with a role for the AT-rich motif in regulating infection-related MtENOD11 expression.

To investigate this in more detail, we analyzed the level of $G U S$ expression for a -257 deletion fusion in which the AATAA sequence has been mutated to CCGCG (Fig. 3). Reporter gene expression associated with rhizobial infection driven by the mutated version of the -257 deletion was reduced significantly by comparison with the nonmutated -257 promoter (Fig. 4). Comparable results were obtained using either a semiquantitative (percentage of GUS-expressing roots) or a quantitative fluorimetric GUS assay (compare Fig. 4A and B). Furthermore, reduced GUS expression resulting from the mutation in the AT-rich motif was also observed in young nodules (data not shown). The mutation of this motif did not significantly modify the nonsymbiotic expression of the -257 promoter fusion in lateral root primordia (data not shown); therefore, these results argue that the AT-rich motif within the first $257 \mathrm{bp}$ of the MtENOD11 promoter indeed plays a role in driving highlevel infection-related gene expression. Analogous experiments were then performed to study the effect of the conversion of the AT-rich motif to a GC-rich sequence on reporter gene expression for both the AM and nematode interactions. The semiquantitative results presented in Figure 4A show that the AT-rich motif mutation results in strongly reduced expres-

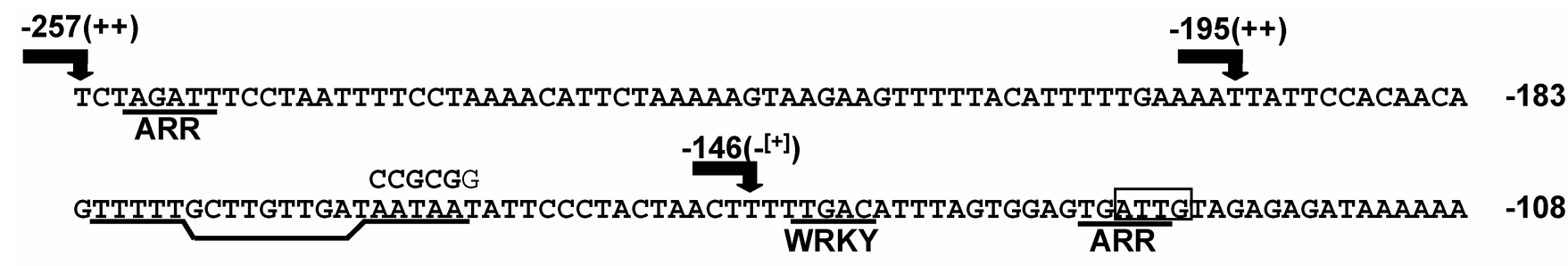

AAAAAAAAAGTTTTGTATTAAGACСTTACACTCCQATTGAGGTCCCAAACTAGTATAAAACCTAAGCATTCCCTA

$+1$

TATGTTATGCACACTAAAATCAСТАССТАAАATG

Fig. 3. The -257 promoter sequence. CAAT and TATA boxes are boxed and double-underlined, respectively. The translation initiation ATG codon is in italics and represents the +1 position with respect to the deletions. The two underlined sequences separated by 10 nucleotides correspond to the putative "weak" (TTTTT) and "strong" (AATAA) AT-rich motifs involved in the expression of early nodulin genes in root nodules according to Hansen and associates (1999). Arrows indicate the positions of the promoter deletion constructs at -195 and -146 positions which showed either similar $(++)$ or strongly reduced $\left(-{ }^{[+]}\right) \beta-$ glucuronidase reporter activities in relation to the -257 deletion $(++)$. The mutated version of the -257 promoter deletion contains, as indicated, a CCGCG substitution for the "strong" AT-rich motif. Core sequences for potential WRKY and ARR1 cis-elements are indicated. 
sion in AM-colonized roots. On the other hand, no difference in expression levels was observed for the mutated construct in root tissues associated with nematode-elicited giant cell differentiation. Taken together, these findings lead us to conclude that, whereas the AT-rich motif appears to play a role in MtENOD11 transcription regulation during the two endosymbiotic associations, this apparently is not the case either for the parasite-root interaction or during lateral root development.

\section{DISCUSSION}

A detailed functional analysis of the MtENOD11 promoter has revealed that distinct promoter regions are involved in preinfection (NF-mediated) expression compared with infectionrelated expression during early stages of the Medicago truncatula-S. meliloti symbiotic association. In this study, we show that a 257-bp region immediately upstream from the MtENOD11 promoter ATG start codon is sufficient to drive infection-related gene expression in both the root epidermis and cortex and in nodule tissues, but is not sufficient to confer early NF-elicited expression in root epidermal cells. Conversely, promoter sequences located further upstream are necessary and sufficient for NF-dependent MtENOD11 expression, but are not sufficient for infection-related expression (A. Andriankaja, A. Boisson-Dernier, D. Barker, and F. de Carvalho-Niebel, unpublished). These results strongly suggest that at least two distinct regulatory pathways are involved in activating MtENOD11 expression during the establishment of the nitrogen-fixing symbiosis.

The possibility of separating regulatory regions within the MtENOD11 promoter has allowed us to investigate gene regulation in the root epidermis in more detail. For example, the $S$. meliloti nodFnodL mutant generates NFs which are modified at the nonreducing end of the lipo-chitooligosaccharide signaling molecule (Ardourel et al. 1994), resulting in a failure to infect the host plant. Nevertheless, purified NFs from the nodFnodL mutant are perfectly capable of activating the rapid NF-dependent expression of MtENOD11 in root hairs (Charron et al. 2004). By using the -257 MtENOD11 promoter deletion, we have been able to study infection-related gene regulation in root hairs without interference from the NF-dependent (preinfection) activation of the gene. In this way, we have been able to show clearly that the -257 promoter region is still transcriptionally activated in root hairs of plants inoculated with the mutant nodFnodL (Fig. 2F). This mutant initiates hair curling but is unable to initiate infection threads; thus, we can deduce that infection thread formation is not a prerequisite for MtENOD11 infection-related expression in root hairs. Furthermore, inoculation with the $S$. meliloti nodA mutant, which is defective in NF production, elicits neither early symbiotic responses such as root hair curling nor expression of the 257bp MtENOD11 promoter. Taken together, these results indicate that i) the activation of the infection-related component of the MtENOD11 promoter depends on the presence of rhizobia producing biologically active NFs, ii) NF alone is not sufficient for infection-related gene activation, and iii) this transcriptional activation precedes infection thread formation. Thus, activation of the 257-bp promoter region requires NF and at least one additional trigger resulting from the presence of the symbiotic bacteria. Bacterial surface envelope polysaccharide signal molecules such as EPS could possibly be part of this secondary activation pathway, because they are required together with NFs for successful bacterial invasion and infection thread formation (Mathis et al. 2005).

Site-directed mutagenesis focused on the AT-rich motif TTATT(N) $)_{7-12}$ AATAA (Hansen et al. 1999) present within the 257-bp truncated MtENOD11 promoter has shown that this
A

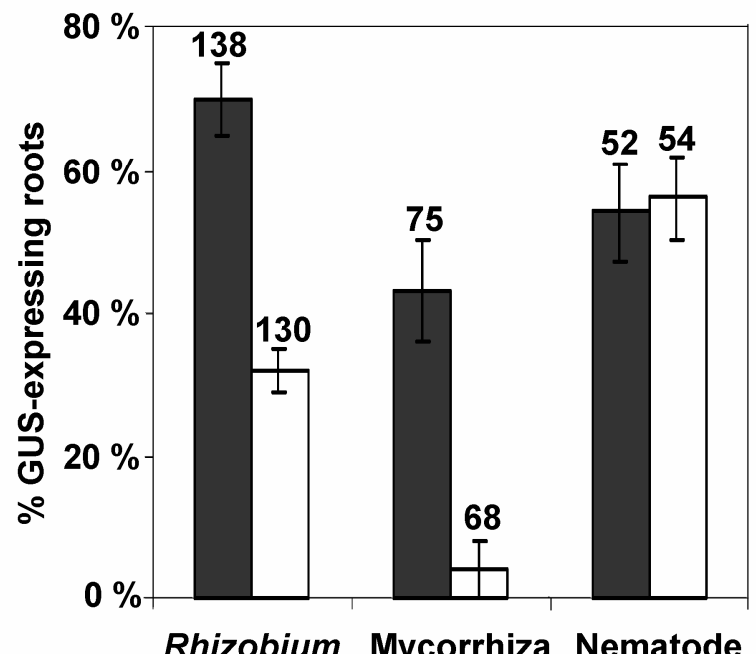

B

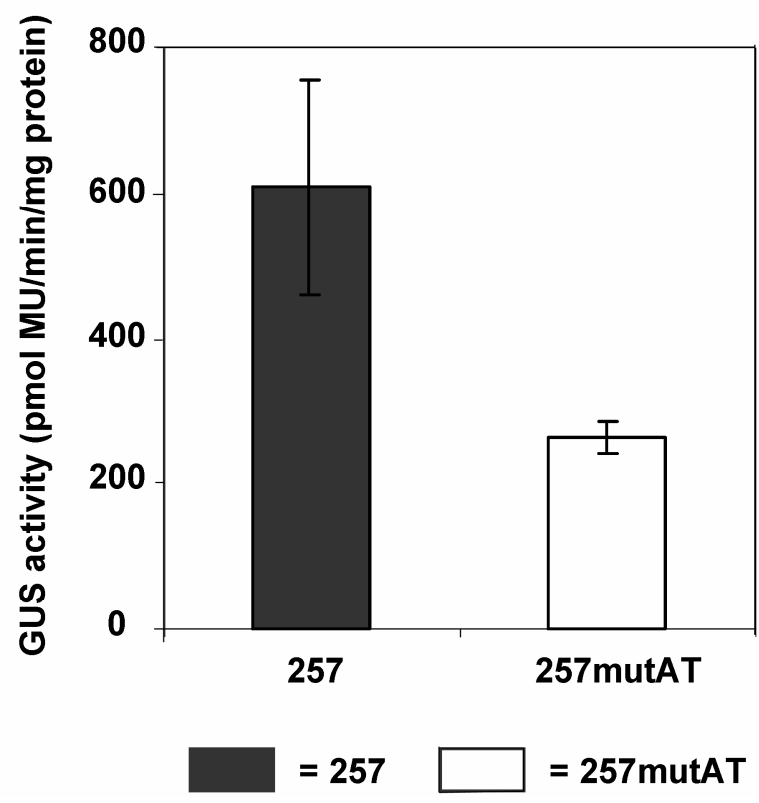

Fig. 4. AATAA motif within the -257 promoter sequence is important for $M t E N O D 11$ expression during both endosymbiotic interactions. A, Semiquantitative analysis of $\beta$-glucuronidase (GUS) activity in roots expressing the -257 MtENOD11 promoter deletion (257) or a mutated version in which the AATAA sequence has been substituted by CCGCG (257mutAT). In all cases, histochemical GUS staining in transgenic roots was evaluated after $6 \mathrm{~h}$ and classified according to the staining intensity based on the method described by Plesch and associates (2001). Only the proportion of independent transgenic roots expressing detectable GUS activity $(++$ and + ) are indicated. Dark and white bars correspond to the nonmutated (257) and mutated (257mutAT) -257 MtENOD11 promoter deletion, respectively. Values are presented as mean percentages of three independent experiments, and the total number of individual transgenic roots analyzed per construct is indicated above the bars. Bars represent \pm standard deviations. Transgenic roots harboring the 257 and the 257mutAT constructs were analyzed 4 days postinoculation with Sinorhizobium meliloti (Rhizobium) 20 days postinoculation with Glomus intraradices (Mycorrhiza) and 5 days postinoculation with Meloidogyne incognita (Nematode). B, Fluorimetric GUS activity measured in transgenic roots transformed with 257 (dark bar) and 257mutAT (white bar) constructs 4 days after $S$. meliloti inoculation. GUS activity levels are mean values of three independent measurements from a total of 85 to 90 independent transgenic roots. Bars represent \pm standard deviations. 
motif is important for infection-related gene expression (Fig. 4). Our analyses of MtENOD11 regulation have further shown that the AT-rich motif is also essential for high-level expression of the 257-bp truncated promoter during the AM-endosymbiotic association, whereas replacement of the major AATAA component by a GC-rich sequence did not significantly affect expression in either lateral root primordia or tissues associated with developing nematode feeding sites. These results suggest that different regulatory pathways are involved in endosymbiotic infection-related transcriptional activation of MtENOD11, compared with expression during the developmental processes of root primordia formation and nematode feeding site initiation and development. In this respect, it is probably significant to note that MtENOD11 expression in nematode feeding sites and in lateral root primordia is associated with pericycle-derived tissues. Likewise, the early nodulin MtENOD40 gene, involved in both early NF-dependent responses and in the regulation of nodule primordia formation, is also expressed in pericycle-derived cells surrounding nematode feeding sites (Favery et al. 2002). It has been suggested that ENOD40 may play a role in cell-to-cell communication events between vascular (giant-cell containing) and surrounding cortical tissues. In the case of the RPRP encoded by MtENOD11 (Journet et al. 2001), function is likely to be related to cell wall modifications involved in the development of specialized feeding cells, as was also proposed for a tobacco extensin (Davis et al. 2004; Niebel et al. 1993). The role of MtENOD11 during endosymbiotic associations with both rhizobia and AM fungi is also likely to be related to specific cell wall and matrix properties required at the plant cell-microsymbiont interface (Journet et al. 2001).

The symbiosis-specific AATAA regulatory sequence described in this study was originally identified by virtue of its role in the expression of the pea ENOD12B gene in Vicia root nodules (Hansen et al. 1999). It was shown further that this AT-rich motif is specifically bound in vitro by a trans-acting factor named VsENBP1, which contains an N-terminal DNAbinding domain featuring several AT-hook motifs typical of certain high-mobility-group (HMG) proteins (Hansen et al. 1999). HMGs are architectural proteins involved in assisting the assembly of functional, higher-order transcription complexes, often by remodeling chromatin structure and thus permitting the activity of neighboring transcription factors (Grasser 2003). Therefore, it is conceivable that chromatinassociated factors are involved in early nodulin gene expression in root or nodule tissues associated with microsymbiont colonization. Our analyses of MtENOD11 regulation have shown that the AT-rich motif is essential for high-level expression of the 257-bp truncated promoter only during rhizobia and AM-endosymbiotic associations. This implies that the ATrich motif and the potential (chromatin-associated) interacting proteins are not simply required for global expression of the

Table 1. Primers used in this study ${ }^{\mathrm{a}}$

\begin{tabular}{ll}
\hline Name & \multicolumn{1}{c}{ Sequence } \\
\hline $5^{\prime}-810$ & 5'-GGGACACGGAATTCTCTTGG -3' \\
5'-720 & 5'-GGGGACAGCCGAATTCATTTACGGAGGTCC-3' \\
5'-528 & 5'-GTGAATGTAAGCTTGTATCTGAGTAATGC-3' \\
5'-411 & 5'-AATAAAGAATTCGACACTTAAATTTGAGGG-3' \\
5'-195 & 5'-GTTTTTACATTTTTGAAGCTTATTCCAC-3' \\
$5^{\prime}-172$ & 5'-GTTGATCCGCGGATTCCCTAC-3' \\
5'-146 & 5'-CCCTACTAAGCTTTTGACATTTAGTG-3' \\
$3^{\prime}-157^{-1}$ & 5'-GAATCCGCGGATCAACAAGC-3' \\
$3^{\prime}-0^{-1}$ & 5'-GGACGGACCATGGTAGGTAGTG-3' \\
$3^{\prime}$-GUS ${ }^{-1}$ & 5'-TGCCCACAGGCCGTCGAG-3' \\
\hline
\end{tabular}

${ }^{a}$ Nucleotides in bold correspond to substitutions in order to generate new restriction sites (underlined). early nodulin gene, but play a role in a specific regulatory pathway related to the symbiotic expression of MtENOD11.

Although several other early nodulin genes have been shown to be regulated both in rhizobial and endomycorrhizal symbioses (Albrecht et al. 1998; Journet et al. 2001; Hohnjec et al. 2003; Manthey et al. 2004; Vieweg et al. 2004, 2005), common endosymbiosis-specific promoter motifs have not been identified thus far. Therefore, the AT-rich motif present in the truncated 257-bp MtENOD11 promoter constitutes the first motif involved in the regulation of gene expression during both endosymbiotic associations. Furthermore, in both cases, expression is specific of root (and nodule) tissues containing cells undergoing intracellular infection or colonization by the respective microsymbiont. The role of the AT-rich motif in endosymbiotic infection-related expression could be to indirectly facilitate the binding of specific transcription factors to neighboring cis-regulatory sequences. Promoter inspection using the PLACE database (Higo et al. 1999) identified certain candidate motifs close to the AT-rich motif (Fig. 3), including W-box and ARR1 motifs, implicated in pathogen and cytokinin signaling pathways, respectively (Rushton et al. 2002; Sakai et al. 2001). We do not yet know whether these motifs have a functional role in regulated expression of MtENOD11, but it is interesting to note that they are also found in the vicinity of the AT-rich motif of the MtENOD12 promoter (Pichon et al. 1992), which is also expressed during endosymbiotic infection or colonization (Pichon et al. 1992) (data not shown).

\section{MATERIALS AND METHODS}

\section{Plant and bacterial strains.}

A17 or J5 M. truncatula Gaertn. cv. Jemalong lines were used in this study. Seed were surface sterilized and germinated on inverted agar plates at $14^{\circ} \mathrm{C}$ in the dark according to BoissonDernier and associates (2001).

ARqual is a streptomycin-resistant derivative of $A$. rhizogenes strain A4T (Quandt et al. 1993). The S. meliloti wild-type strain RCR2011 (GMI6526) and mutant strains nodFnodL (GMI6630) and nodA (GMI6702) used in this study carry a constitutive hemA-lacZ fusion (Ardourel et al. 1994). A. rhizogenes and $S$. meliloti strains were grown at $28^{\circ} \mathrm{C}$ in tryptone yeast medium with the appropriate antibiotics.

\section{Generation of $A$. rhizogenes strains} containing $\mathbf{5}^{\prime}$ promoter deletion constructs.

Construction of the 2.3-kb pMtENOD11-GUS fusion in the pLP100 binary vector has been described by Charron and associates (2004). Using internal EcoRI, BglII, StuI, and XbaI restriction sites within the full-length $2.3-\mathrm{kb}$ MtENOD11 promoter, we generated deletion constructs $-998,-882,-367$, and -257 , respectively. Promoter fragments for the remaining deletion constructs $(-810,-720,-528,-411,-195$, and -146) were generated by polymerase chain reaction (PCR) using the pMtENOD11-GUS containing plasmid as a template. PCR amplification was performed with $5^{\prime}$ primers covering the respective deletion positions (Table 1) in combination with the T7 universal primer as the $3^{\prime}$ primer for constructs $-810,-720,-528$, and -411 , and the $3^{\prime}$ primer $0^{-1}$ overlapping the ATG start codon (Table 1) for constructs -195 and -146 . Substitution of the AT-rich motif ( -166 to -162 positions) within the -257 deletion construct was generated by introduction of a SacII restriction site within this motif. For this purpose, we generated two PCR fragments corresponding to the -411 to -157 and the -172 to +1 promoter regions by using the $5^{\prime}-411$ and $3^{\prime}-157^{-1}$ and $5^{\prime}-172$ and $3^{\prime} 0^{-1}$ primer pairs, respectively. These two PCR fragments containing the XbaI/SacII and SacII/NcoI restriction sites, respectively, at their ends, were ligated together 
to generate the AT-rich motif mutation in the -257 deletion. After restriction digestion, the ligated PCR fragments were subcloned between the $\mathrm{XbaI}$ and $\mathrm{NcoI}$ sites of a pUC19 derivedvector to produce the $-G U S$ fusion construct. Sequencing using a $3^{\prime} G U S^{-1}$ primer was performed on all constructs to confirm PCR fidelity. The corresponding deletion constructs subcloned into the binary vector pLP100 were then introduced into $A$. rhizogenes strain ARqual by the freeze-thaw method as described in Höfgen and Willmitzer (1988). Kanamycin-resistant $\left(\mathrm{Km}^{\mathrm{r}}\right)$ ARqua1 transformants were reanalyzed by PCR to confirm the presence of the transgene of interest before plant transformation.

\section{Transformation of M. truncatula with $A$. rhizogenes and $S$. meliloti inoculation and Nod factor treatments.}

$M$. truncatula roots were transformed with the various constructs using the $A$. rhizogenes technique as described by Boisson-Dernier and associates (2001). At approximately 3 weeks after inoculation by $A$. rhizogenes, composite $M$. truncatula plants (wild-type aerial part with transgenic roots) were transferred to pouch-paper (Mega International, Minneapolis, MN, U.S.A.) laying on Fahraeus agar plates (without nitrogen or $\mathrm{Km}$ ). This system was found to be very efficient for nodulation. Composite $M$. truncatula plant roots grown in this way were flood-inoculated with either an $S$. meliloti suspension $(5 \times$ $10^{5}$ bacteria/ml) or an NF aqueous solution $\left(10^{-8}\right.$ to $\left.10^{-9} \mathrm{M}\right)$.

\section{Analyses of mycorrhiza and nematode-inoculated composite $M$. truncatula plants.}

For mycorrhizal inoculation, agar plates containing $G$. intraradices-colonized in vitro-grown carrot (Daucus carota) $\mathrm{Ri}$ transformed roots were ground gently with sterile water and the resulting mixture was spread on composite $M$. truncatula plant roots grown on pouch-paper laid on $0.5 \%$ phytagel (SigmaAldrich, St Louis, U.S.A.) containing M medium (Bécard and Fortin 1988). For each construct, approximately 70 independent mycorrhized hairy roots were harvested 3 to 4 weeks postinoculation and analyzed for transgene expression. Staining of fungal structures inside mycorrhized roots was performed as described by Chabaud and associates (2002). For nematode infection, Meloidogyne incognita infective juvenile nematodes were rinsed and resuspended in sterile water before deposition on root tips of composite Medicago truncatula plants grown on pouch-paper laid on Fahraeus-agar plates (Boisson-Dernier et al. 2001). For each construct, at least 50 independent nematode-infected hairy roots were harvested 5 dpi for transgene expression analysis. For nematode detection, infected roots were stained for $16 \mathrm{~h}$ with an acidic fuchsin solution $(17.5 \mathrm{mg} /$ liter in $1: 1$ volume of ethanol $95 \%$ and glacial acetic acid) following by 24 of $36 \mathrm{~h}$ of incubation in sterile water.

\section{Histochemical and fluorimetric GUS assays.}

Histochemical GUS staining was performed essentially as described previously (Journet et al. 2001), by using the XglcUA (5-bromo-4-chloro-3-indolyl glucuronide, cyclohexylammonium salt) (Biosynth, Staad, Switzerland) or MagentaglcUA (Biosynth) substrates to obtain blue- or red-colored GUS staining, respectively. Stained samples were observed with a stereomicroscope (Leica Microsystems, Wetzler, Germany), a Zeiss Axiophot light microscope (Carl Zeiss, Oberkochen, Germany), or both. Double staining for both GUS and $\beta$-galactosidase activities after inoculation with $S$. meliloti strains carrying a constitutive hemA-lacZ fusion (Ardourel et al. 1994) was performed according to Vernoud and associates (1999). Nodule sections (80- $\mu \mathrm{m}$ thick) were also prepared as described by Vernoud and associates (1999).
To evaluate GUS staining intensities for different promoter deletion constructs, stained roots were analyzed at the following timepoints post-staining: $30 \mathrm{~min}, 1 \mathrm{~h} 30 \mathrm{~min}, 3 \mathrm{~h}, 6 \mathrm{~h}$, and $15 \mathrm{~h}$. For simplicity, overall GUS staining was classified into the three following categories: clear-cut expression $(+)$, residual very weak expression $\left(-^{[+]}\right)$and undetectable expression (-). For scoring the -257 deletion and the related construct mutated in the AT-rich motif, we performed an alternative evaluation of GUS intensity based on a semiquantitative method developed by Plesch and associates (2001). For each deletion, 130 independent roots from three separate experiments were scored for undetectable $(-)$, weak $(+)$, and moderate (++) GUS intensities.

Fluorimetric GUS assays were performed as described previously (Jefferson 1987). In all, 17 to 18 pools of five independent hairy roots were ground in liquid nitrogen and homogenized in GUS buffer ( $50 \mathrm{mM}$ sodium phosphate buffer, $\mathrm{pH} 7.5$; 10 mM 2-mercaptoethanol; 10 mM EDTA; 0.1\% Triton X100; and $0.1 \%$ sodium lauryl-sarcosine) for total protein extraction. Enzymatic reactions were carried out using $1 \mu \mathrm{g}$ of total protein extract with 4-methylumbelliferyl- $\beta$-D-glucuronide as substrate (Biosynth AG, Geneva, Switzerland). GUS activities were measured using a microtiter fluorimeter (FL600, Bio-Tek, Highland Park, VT, U.S.A.). Standard curves were prepared with a range of increasing concentrations of 4-methylumbelliferone (Sigma-Aldrich).

\section{ACKNOWLEDGMENTS}

We would like to thank J.-M. Prospéri (INRA, Montpellier) and J. Garcia for M. truncatula A17 seed, F. Maillet and J. Denarié for providing purified Nod factors, S. Jaubert and B. Favery from P. Abad's laboratory (INRA, Sophia Antipolis, Nice) and S. Roy (Université Paul Sabatier/ CNRS, Toulouse) for kindly providing $M$. incognita L2 juvenile nematodes and $G$. intraradices spores, respectively.

\section{LITERATURE CITED}

Albrecht, C., Guerts, R., Lapeyrie, F., and Bisseling, T. 1998. Endomycorrhizae and rhizobial Nod factors both require $S Y M 8$ to induce the expression of the early nodulin genes PsENOD5 and PsENOD12A. Plant J. 15:605-614.

Andersson, C. R., Llewellyn, D. J., Peacock, W. J., and Dennis, E. S. 1997. Cell-specific expression of the promoters of two nonlegume hemoglobin genes in a transgenic legume, Lotus corniculatus. Plant Physiol. 113:45-57.

Ardourel, M., Demont, N., Debelle, F., Maillet, F., de Billy, F., Prome, J. C., Denarie, J., and Truchet, G. 1994. Rhizobium meliloti lipooligosaccharide nodulation factors: Different structural requirements for bacterial entry into target root hair cells and induction of plant symbiotic developmental responses. Plant Cell 6:1357-1374.

Bécard, G., and Fortin, J. A. 1988. Early events of vesicular-arbuscular mycorrhiza formation on Ri T-DNA transformed roots. New Phytol. 108:211-218.

Bird, D. M. 2004. Signaling between nematodes and plants. Curr. Opin. Plant Biol. 7:372-376.

Boisson-Dernier, A., Chabaud, M., Garcia, F., Becard, G., Rosenberg, C., and Barker, D. G. 2001. Agrobacterium rhizogenes-transformed roots of Medicago truncatula for the study of nitrogen-fixing and endomycorrhizal symbiotic associations. Mol. Plant-Microbe Interact. 14:695-700.

Catoira, R., Galera, C., de Billy, F., Penmetsa, R. V., Journet, E. P., Maillet, F., Rosenberg, C., Cook, D., Gough, C., and Denarie, J. 2000. Four genes of Medicago truncatula controlling components of a nod factor transduction pathway. Plant Cell 12:1647-1666.

Chabaud, M., Venard, C., Defaux-Petras, A., Bécard, G., and Barker, D. 2002. Target inoculation of Medicago truncatula in vitro root cultures reveals $M t E N O D 11$ expression during early stages of infection by arbuscular mycorrhizal fungi. New Phytol. 156:265-273.

Charron, D., Pingret, J. L., Chabaud, M., Journet, E. P., and Barker, D. G. 2004. Pharmacological evidence that multiple phospholipid signaling pathways link Rhizobium nodulation factor perception in Medicago truncatula root hairs to intracellular responses, including $\mathrm{Ca}^{2+}$ spiking and specific ENOD gene expression. Plant Physiol. 136:3582-3593. 
Davis, E. L., Hussey, R. S., and Baum, T. J. 2004. Getting to the roots of parasitism by nematodes. Trends Parasitol. 20:134-141.

D'Haeze, W., and Holsters, M. 2002. Nod factor structures, responses, and perception during initiation of nodule development. Glycobiology 12:79R-105R.

Favery, B., Complainville, A., Vinardell, J. M., Lecomte, P., Vaubert, D., Mergaert, P., Kondorosi, A., Kondorosi, E., Crespi, M., and Abad, P. 2002. The endosymbiosis-induced genes ENOD40 and CCS52a are involved in endoparasitic-nematode interactions in Medicago truncatula. Mol. Plant-Microbe Interact. 15:1008-1013.

Gage, D. J. 2004. Infection and invasion of roots by symbiotic, nitrogenfixing rhizobia during nodulation of temperate legumes. Microbiol. Mol. Biol. Rev. 68:280-300.

Grasser, K. D. 2003. Chromatin-associated HMGA and HMGB proteins: Versatile co-regulators of DNA-dependent processes. Plant Mol. Biol. 53:281-295.

Hansen, A. C., Busk, H., Marcker, A., Marcker, K. A., and Jensen, E. O. 1999. VsENBP1 regulates the expression of the early nodulin PsENOD12B. Plant Mol. Biol. 40:495-506.

Higo, K., Ugawa, Y., Iwamoto, M., and Korenaga, T. 1999. Plant cis-acting regulatory DNA elements (PLACE) database: 1999. Nucleic Acids Res. 27:297-300.

Höfgen, R., and Willmitzer, L. 1988. Storage of competent cells for Agrobacterium transformation. Nucleic Acids Res. 16:9877.

Hohnjec, N., Perlick, A. M., Puhler, A., and Kuster, H. 2003. The Medicago truncatula sucrose synthase gene MtSucS1 is activated both in the infected region of root nodules and in the cortex of roots colonized by arbuscular mycorrhizal fungi. Mol. Plant-Microbe Interact. 16:903-915.

Jefferson, R. A., Kavanagh, T. A., and Bevan, M. W. 1987. GUS fusions: Beta-glucuronidase as a sensitive and versatile gene fusion marker in higher plants. EMBO (Eur. Mol. Biol. Organ.) J. 6:3901-3907.

Journet, E. P., El-Gachtouli, N., Vernoud, V., de Billy, F., Pichon, M., Dedieu, A., Arnould, C., Morandi, D., Barker, D. G., and GianinazziPearson, V. 2001. Medicago truncatula ENOD11: A novel RPRP-encoding early nodulin gene expressed during mycorrhization in arbusculecontaining cells. Mol. Plant-Microbe Interact. 14:737-748.

Kistner, C., and Parniske, M. 2002. Evolution of signal transduction in intracellular symbiosis. Trends Plant Sci. 7:511-518.

Koltai, H., Dhandaydham, M., Opperman, C., Thomas, J., and Bird, D. 2001. Overlapping plant signal transduction pathways induced by a parasitic nematode and a rhizobial endosymbiont. Mol. Plant-Microbe Interact. 14:1168-1177.

Limpens, E., and Bisseling, T. 2003. Signaling in symbiosis. Curr. Opin. Plant Biol. 6:343-350.

Manthey, K., Krajinski, F., Hohnjec, N., Firnhaber, C., Puhler, A., Perlick, A. M., and Kuster, H. 2004. Transcriptome profiling in root nodules and arbuscular mycorrhiza identifies a collection of novel genes induced during Medicago truncatula root endosymbioses. Mol. Plant-Microbe Interact. 17:1063-1077.

Mathis, R., Van Gijsegem, F., De Rycke, R., D’Haeze, W., Van Maelsaeke, E., Anthonio, E., Van Montagu, M., Holsters, M., and Vereecke, D. 2005. Lipopolysaccharides as a communication signal for progression of legume endosymbiosis. Proc. Natl. Acad. Sci. U.S.A. 102:2655-2660.

Niebel, A., De Almeida Engler, J., Tire, C., Engler, G., Van Montagu, M., and Gheysen, G. 1993. Induction patterns of an extensin gene in tobacco upon nematode infection. Plant Cell 5:1697-1710.

Oldroyd, G. E., Harrison, M. J., and Udvardi, M. 2005. Peace talks and trade deals. Keys to long-term harmony in legume-microbe symbioses. Plant Physiol. 137:1205-1210.

Pichon, M., Journet, E. P., Dedieu, A., de Billy, F., Truchet, G., and Barker, D. G. 1992. Rhizobium meliloti elicits transient expression of the early nodulin gene ENOD12 in the differentiating root epidermis of transgenic alfalfa. Plant Cell 4:1199-1211.

Plesch, G., Ehrhardt, T., and Mueller-Roeber, B. 2001. Involvement of TAAAG elements suggests a role for Dof transcription factors in guard cell-specific gene expression. Plant J. 28:455-464.

Quandt, H. J., Puhler, A., and Broer, I. 1993. Transgenic root nodules of Vicia hirsuta: A fast and efficient system for the study of gene expression in indeterminate-type nodules. Mol. Plant-Microbe Interact. 6:699-706.

Rodriguez-Llorente, I. D., Pérez-Hormaeche, J., Dary, M., Caviedes, A M., Trinh, T. H., Kondorosi, A., Ratet, P., and Palomares, A. J. 2003. $M s P G 3$ polygalacturonase promoter elements necessary for expression during Sinorhizobium meliloti-Medicago truncatula interaction. Plant Soil 257:19-26.

Rushton, P. J., Reinstadler, A., Lipka, V., Lippok, B., and Somssich, I. E. 2002. Synthetic plant promoters containing defined regulatory elements provide novel insights into pathogen- and wound-induced signaling. Plant Cell 14:749-762.

Sakai, H., Honma, T., Aoyama, T., Sato, S., Kato, T., Tabata, S., and Oka A. 2001. ARR1, a transcription factor for genes immediately responsive to cytokinins. Science 294:1519-1521.

Stougaard, J., Sandal, N. N., Gron, A., Kühle, A., and Marcker, K. A. 1987. 5' analysis of the soybean leghaemoglobin $l b c_{3}$ gene: Regulatory elements required for promoter activity and organ specificity. EMBO (Eur. Mol. Biol. Organ.) J. 6:3565-3569.

Vernoud, V., Journet, E. P., and Barker, D. G. 1999. MtEnod20, a nod factor-inducible molecular marker for root cortical cell activation. Mol. Plant-Microbe Interact. 12:604-614.

Vieweg, M. F., Fruhling, M., Quandt, H. J., Heim, U., Baumlein, H., Puhler, A., Kuster, H., and Andreas, M. P. 2004. The promoter of the Vicia faba L. leghemoglobin gene VfLb29 is specifically activated in the infected cells of root nodules and in the arbuscule-containing cells of mycorrhizal roots from different legume and nonlegume plants. Mol. Plant-Microbe Interact. 17:62-69.

Vieweg, M. F., Hohnjec, N., and Kuster, H. 2005. Two genes encoding different truncated hemoglobins are regulated during root nodule and arbuscular mycorrhiza symbioses of Medicago truncatula. Planta 220:757-766.

Vijn, I., Christiansen, H., Lauridsen, P., Kardailsky, I., Quandt, H. J., Broer, I., Drenth, J., Ostergaard Jensen, E., van Kammen, A., and Bisseling, T. 1995. A 200 bp region of the pea ENOD12 promoter is sufficient for nodule-specific and nod factor induced expression. Plant Mol. Biol. 28:1103-1110.

\section{AUTHOR-RECOMMENDED INTERNET RESOURCE}

PLACE database: www.dna.affrc.go.jp/htdocs/PLACE 